\title{
COMPOSIÇÃO QUÍMICA E ATIVIDADE ANTILEISHMANIA DE Tocoyena hispidula
}

Elcilene A. de Sousa ${ }^{a}$, Amauri Porto A. Rosa ${ }^{a}$, Rodolfo Ritchelle L. dos Santos ${ }^{b}$ Ingredy L. dos Santos ${ }^{b}$, Valéria C. de Sousa ${ }^{b}$, Fernando Aécio de A. Carvalho ${ }^{b}$, Gerardo M. Vieira Júnior ${ }^{a}$ e Mariana H. Chaves ${ }^{\text {a,* }}$

aDepartamento de Química, Universidade Federal do Piauí, 64049-550 Teresina - PI, Brasil

bepartamento de Bioquímica e Farmacologia, Universidade Federal do Piauí, 64049-550 Teresina - PI, Brasil

Recebido em 15/09/2018; aceito em 07/11/2018; publicado na web em 28/11/2018

\begin{abstract}
CHEMICAL COMPOSITION AND ANTI-LEISHMANIA ACTIVITY FROM Tocoyena hispidula. Phytochemical investigation of the $\mathrm{CHCl}_{3}$ fraction from EtOH extract of Tocoyena hispidula (Rubiaceae) stem resulted in the isolation and identification of D-(+)-mannitol, lupenone, 3-O-acetyloleanolic acid, lapachol, dimethyl chelidonate, morindolide and four mixtures (M1-M4): M1 (palmitate, margarate, linoleate, oleate e stearate of the multiflorenyl, lupeyl, sitosteryl and stigmasteryl), M2 (lupeol, taraxerol, germanicol, $\beta$-amyrin and $E$-fitol), $M 3$ (campesterol, campestanol, stigmasterol, $\Delta^{22}$-stigmastenol, sitosterol and sitostanol) and M4 (7-ketositosterol and 7-ketostigmasterol). Structural identification of the compounds was performed by analysis ${ }^{1} \mathrm{H}$ and ${ }^{13} \mathrm{C}$ NMR spectra and by GC-MS. Extract, fractions, dimethyl chelidonate and morindolide inhibited the growth of Leishmania major promastigotes, being the $\mathrm{CHCl}_{3}\left(\mathrm{IC}_{50}=26.25 \mu \mathrm{g} \mathrm{mL}^{-1}\right)$ and EtOAc $\left(\mathrm{IC}_{50}=29.77 \mu \mathrm{g} \mathrm{mL} \mathrm{m}^{-1}\right)$ fractions the more active.
\end{abstract}

Keywords: chemical composition; Tocoyena hispidula; Rubiaceae; anti-Leishmania.

\section{INTRODUÇÃO}

Rubiaceae é considerada, entre as Angiospermas, a quarta maior família com 650 gêneros e 13.000 espécies. ${ }^{1}$ É caracterizada como uma das mais importantes da flora brasileira, com 126 gêneros e 1.412 espécies. ${ }^{2,3}$ É constituída pelas subfamílias Rubioideae, Ixoroideae e Cinchonoideae, as quais possuem como marcadores quimiotaxonômicos antraquinonas, iridoides e alcaloides indólicos, respectivamente. Plantas da família Rubiaceae são também conhecidas por acumularem triterpenoides, saponinas, diterpenoides, esteroides, cumarinas, flavonoides, lignanas, derivados fenólicos e taninos, sendo os triterpenoides amplamente distribuídos em todas as subfamílias. ${ }^{4}$

Diversas atividades biológicas têm sido relatadas em plantas da família Rubiaceae tais como, anti-inflamatória, analgésica, antiviral, antibacteriana, antioxidante, antifúngica, antimicrobiana, anticâncer, anti-helmíntica, antidiabética, antimalárica, hepatoprotetora e antileishmania..$^{5,6}$

O gênero Tocoyena (Rubiaceae) é típico do Cerrado, ocorre na região neotropical, sendo constituído por 30 espécies de hábito arbustivo ou árvores de pequeno porte. No Brasil há registro de 12 espécies, sendo cinco endêmicas, distribuídas em todas as regiões brasileiras. ${ }^{7}$ Esse gênero pertence à subfamília Ixoroideae, tribo Gardenieae-Gardenineae. ${ }^{1,8}$ Somente quatro espécies de Tocoyena possuem estudo químico (T. formosa, T. bullata, T. brasiliensis e T. sellowiana, sinonímia $T$. selloana), para as quais são relatadas a presença de iridoides, flavonoides, esteroides, cumarinas, derivados fenólicos, triterpenoides e saponinas triterpênicas. ${ }^{8-10}$ Algumas atividades biológicas têm sido relatadas, como anti-inflamatória, antinociceptiva, antifúngica, estimulante em útero de ratas, antioxidante e gastroprotetora para $T$. formosa e capacidade de diminuição da perda óssea em ratos com periodontite para T. sellowiana. ${ }^{9,11}$

Tocoyena hispidula Standl é conhecida popularmente por flor-do-cerrado/angelca e jenipapinho, sendo encontrada nas regiões Norte (Pará) e Nordeste (Maranhão e Piauí) do Brasil na forma de arbustos ou subarbustos. Tradicionalmente suas raízes são utilizadas

*e-mail: mariana@ufpi.edu.br no combate a dor de barriga e inflamação do útero, ${ }^{12}$ entretanto, não há registros de estudo químico e biológico sobre esta espécie.

$\mathrm{O}$ presente trabalho relata o isolamento e identificação estrutural de constituintes químicos da fração $\mathrm{CHCl}_{3}$ do extrato etanólico do caule de T. hispidula, bem como a avaliação do seu potencial antileishmania.

\section{PARTE EXPERIMENTAL}

\section{Procedimentos experimentais gerais}

Os espectros de RMN foram obtidos em espectrômetros Varian INOVA-modelo 400 (LAUREMN/UFPI), operando a $400 \mathrm{MHz}\left({ }^{1} \mathrm{H}\right)$ e $100 \mathrm{MHz}\left({ }^{13} \mathrm{C}\right)$ e Bruker Ascend-modelo 600 operando a $600 \mathrm{MHz}$ $\left({ }^{1} \mathrm{H}\right)$ e $150 \mathrm{MHz}\left({ }^{13} \mathrm{C}\right)$. Foram utilizados $\mathrm{CDCl}_{3}$ ou DMSO- $d_{6}$ como solventes e TMS como referência interna. $\mathrm{O}$ espectro de absorção na região do infravermelho (IV) foi registrado em espectrômetro PerkinElmer, modelo Spectrum 100FT-IR, com faixa de número de onda de $4000 \mathrm{a} 400 \mathrm{~cm}^{-1}$. O ponto de fusão foi obtido em equipamento da Microquímica Ind. e Com. Ltda, modelo MQAPF-301, com taxa de aquecimento de $1{ }^{\circ} \mathrm{C} \mathrm{min}{ }^{-1}$. A rotação específica foi obtida em polarímetro Automático Digital da Jasco-Modelo P2000 (Fonte: $\mathrm{Hg}, \lambda=546 \mathrm{~nm})$.

A análise dos esteroides e dos ésteres metílicos por CG-EM foi realizada em cromatógrafo GCMS-QP2010 (Shimadzu), equipado com coluna Rtx-5MS (30 m x 0,25 mm x 0,250 $\mu \mathrm{m}$ ), fase estacionária difenildimetilpolissiloxano (5\% de difenil e $95 \%$ de dimetilpolissiloxano), usando modo split (100:1) e espectrômetro de massas operando com ionização por elétrons (EI $70 \mathrm{eV}$ ) com faixa de massas $\mathrm{m} / z, 45$ a $700 \mathrm{Da}$, utilizando hélio como gás de arraste, com fluxo de $1 \mathrm{~mL} \min ^{-1}$. As condições de operação da análise cromatográfica dos esteroides foram: temperatura inicial do forno de $100{ }^{\circ} \mathrm{C}$ por 2 minutos, com taxa de aquecimento de $10{ }^{\circ} \mathrm{C} \mathrm{min}-1$ até $300{ }^{\circ} \mathrm{C}$ permanecendo por 10 minutos. A temperatura do injetor foi de $280{ }^{\circ} \mathrm{C}$, da interface $290{ }^{\circ} \mathrm{C}$ e da fonte $270{ }^{\circ} \mathrm{C}$. Para os ésteres metilícos foi utilizada a seguinte programação: temperatura inicial do forno de $70^{\circ} \mathrm{C}$ por 2 minutos, com taxa de aquecimento de $6{ }^{\circ} \mathrm{C} \mathrm{min}{ }^{-1}$ até $310^{\circ} \mathrm{C}$, permanecendo por 10 minutos. A temperatura do injetor 
foi de $300{ }^{\circ} \mathrm{C}$, da interface $310{ }^{\circ} \mathrm{C}$ e da fonte $260{ }^{\circ} \mathrm{C}$. Os compostos foram identificados por comparação dos espectros de massas obtidos com os da biblioteca Wiley229.

As placas cromatográficas foram preparadas utilizando uma suspensão de gel de sílica 60 da Macherey-Nagel em água destilada e as revelações das cromatoplacas foram feitas por nebulização com solução de $\mathrm{Ce}\left(\mathrm{SO}_{4}\right)_{2}$. Os solventes e reagentes utilizados na preparação dos extratos e fracionamentos foram todos de pureza analítica (P.A.) obtidos da Synth. Nas separações por cromatografia em coluna foram empregados, gel de sílica 0,060-0,200 mm da Macherey-Nagel e Sephadex LH-20 da Aldrich.

\section{Material vegetal}

O caule de Tocoyena hispidula Standl foi coletado na fazenda Lourdes (S $04^{\circ}$ 51' 32,4" W 42 03 ' 42,9", altitude: $154 \mathrm{~m}$ ), no município de Jatobá do Piauí-PI, em 31 de maio de 2015. O material vegetal foi identificado pela botânica Dra. Ruth Raquel Soares de Farias e, uma exsicata encontra-se depositada no Herbário Graziela Barroso da UFPI com o número TEPB 30.572.

\section{Isolamento dos constituintes químicos}

O caule seco e moído $(1,4 \mathrm{~kg})$ foi submetido à maceração exaustiva com etanol (95\%). O material obtido foi concentrado em evaporador rotativo e liofilizado, fornecendo $48 \mathrm{~g}(3,5 \%) \mathrm{de}$ extrato EtOH. Uma alíquota de $41 \mathrm{~g}$ do extrato foi solubilizada em $\mathrm{MeOH}$, ocorrendo a formação de um precipitado, identificado como o composto 1 (4,5 g; 9,4\%). A parte solúvel em $\mathrm{MeOH}$ do extrato $(36,5 \mathrm{~g})$ foi submetida à cromatografia em coluna filtrante de gel de sílica, utilizando hexano, $\mathrm{CHCl}_{3}$, AcOEt e $\mathrm{MeOH}$ como eluentes. A fração $\mathrm{CHCl}_{3}(2,8 \mathrm{~g})$ foi aplicada em coluna cromatográfica de gel de sílica, eluída com hexano/AcOEt em ordem crescente de polaridade. Foram coletadas 82 frações e reunidas em 16 grupos após análise por CCDC. O grupo CC9 $(340,7 \mathrm{mg})$ foi submetido à cromatografia em coluna de Sephadex LH-20 utilizando hexano/ $\mathrm{CH}_{2} \mathrm{Cl}_{2}$ (1:4) e cromatografia em coluna de gel de sílica, eluída com hexano/AcOEt 98:2, fornecendo a subfração CC9-7-2 (161,2 mg) e o composto 2 (14,3 $\mathrm{mg})$.

Uma alíquota de $50 \mathrm{mg}$ da subfração CC9-7-2 (M1) foi submetida à reação de hidrólise alcalina, ${ }^{13}$ fornecendo, após extração com éter etílico, as fases etérea (FE; 25,2 mg) e aquosa (FA). A fase etérea (FE) foi aplicada em coluna cromatográfica de gel de sílica utilizando hexano/AcOEt 95:5, resultando nas frações FE-21 (2,7 mg) e FE-35 (10,4 $\mathbf{m g}$ ), identificadas como as misturas de $\mathbf{3 + 4}$ e $\mathbf{5 + 6}$, respectivamente. A fase aquosa (FA) foi acidificada com $\mathrm{H}_{2} \mathrm{SO}_{4}$ a $30 \%(\mathrm{pH}=4)$, extraída com éter etílico e metilada com diazometano, ${ }^{13}$ resultando em $10 \mathrm{mg}$ da mistura dos ésteres metílicos dos ácidos palmítico (a: 16:0, 33,72\%), margárico (b: 17:0, 2,50\%), linoleico (c: 18:2, $\Delta^{9,12}$, 22,11\%), oleico (d: $18: 1, \Delta^{9}, 29,90 \%$ ) e esteárico (e: 18:0, 8,52\%), identificados após análise por CG-EM (Figuras 12S-17S).

Os grupos CC13 (143,9 mg), CC15 (167,5 mg) e CC24 (321,0 $\mathrm{mg}$ ) foram aplicados em colunas cromatográficas de Sephadex LH-20, utilizando hexano/ $\mathrm{CH}_{2} \mathrm{Cl}_{2}$ (1:4). Os grupos $\mathrm{CC} 13$ e $\mathrm{CC} 15$ forneceram o composto 18 (44 mg) e a mistura $M 2(\mathbf{4 + 7 + 8 + 9 + 1 1 ; ~}$ $17,7 \mathrm{mg})$, respectivamente, enquanto $\mathrm{CC} 24$ resultou na mistura $M 3$ $(\mathbf{5 + 6 + 1 2 + 1 3 + 1 4 + 1 5 ; 1 1 5 , 0 ~ m g ) ~ e ~ n o ~ c o m p o s t o ~} \mathbf{1 0}(25,9 \mathrm{mg})$. O grupo CC50 (98,0 mg) também foi submetido a cromatografia em coluna de Sephadex LH-20, utilizando hexano/ $\mathrm{CH}_{2} \mathrm{Cl}_{2}$ (1:4), seguido de filtração em gel de sílica com $\mathrm{CHCl}_{3}$, conduzindo à mistura $M 4(\mathbf{1 6 + 1 7} ; 5,4$ $\mathrm{mg}$ ) e ao composto 19 (5,7 mg). O grupo CC72 (100,7 mg), depois de submetido a cromatografia em coluna de Sephadex LH-20, utilizando hexano/ $\mathrm{CH}_{2} \mathrm{Cl}_{2}$ (1:4) forneceu o composto $\mathbf{2 0}$ (46 mg).
Chelidonato de metila (19). Sólido amorfo laranja. PF: $>200{ }^{\circ} \mathrm{C}$ (decomposição). RMN de ${ }^{1} \mathrm{He}{ }^{13} \mathrm{C}(400$ e $100 \mathrm{MHz}$, respectivamente, em $\mathrm{CDCl}_{3}$ ): ver Tabela 1 .

\section{Avaliação da atividade antileishmania}

As formas promastigotas de Leishmania major em fase logarítimica de crescimento foram semeadas em placas de cultivo celular com 96 poços ( 1 x $10^{6}$ leishmania por poço), contendo meio Schneider's suplementado. $\mathrm{O}$ extrato EtOH, frações $\mathrm{CHCl}_{3}, \mathrm{AcOEt}$ e $\mathrm{MeOH}$ e os compostos 19 e $\mathbf{2 0}$ foram adicionados aos poços em diluições seriadas de 800 a $6,25 \mu \mathrm{g} \mathrm{mL} \mathrm{m}^{-1}$. As placas foram incubadas em estufa de demanda biológica (BOD) à temperatura de $26^{\circ} \mathrm{C}$ e, após 48 h, os parasitas foram corados com resazurina $\left(1 \mathrm{mmol} \mathrm{L}^{-1}\right)$ e feita a leitura em espectofotômetro para obtenção da densidade ótica a $550 \mathrm{~nm}$. O controle positivo consistiu de $2 \mu \mathrm{g} \mathrm{mL}^{-1}$ de anfotericina B diluída em meio Schneider's a 0,2\% de DMSO e considerado como $100 \%$ de viabilidade das leishmanias. ${ }^{14}$

\section{RESULTADOS E DISCUSSÃO}

O extrato EtOH do caule de T. hispidula após suspensão em $\mathrm{MeOH}$ formou um precipitado identificado como D-(+)-manitol (1) ${ }^{15} \mathrm{O}$ fracionamento cromatográfico do sobrenadante do extrato EtOH forneceu as frações hexânica (50 mg; 0,15\%), $\mathrm{CHCl}_{3}(2,8 \mathrm{~g}$; $7,70 \%)$, AcOEt (3,6 g; 9,90\%) e MeOH (26,6 g; 72,90\%). A fração $\mathrm{CHCl}_{3}$, depois de submetida a procedimentos cromatográficos clássicos, resultou no isolamento e identificação da lupenona (2), ${ }^{16}$ ácido acetiloleanólico (10), ${ }^{17}$ lapachol (18), ${ }^{18}$ chelidonato de metila (19), morindolídeo (20) ${ }^{19}$ e quatro misturas (M1-M4) de isoprenoides. A mistura $M 1$ é constituída por triterpenoides e esteroides esterificados com ácidos graxos, entre os quais, o palmitato, margarato, linoleato, oleato e estearato de multiflorenila (3a-3e), lupeila (4a4e), sitosterila (5a-5e) e estigmasterila $(\mathbf{6 a - 6 e}) \cdot{ }^{20,21} \mathrm{~A}$ mistura $M 2$ é composta pelos triterpernoides pentacíclicos lupeol (4), taraxerol (7), germanicol (8), $\beta$-amirina $(\mathbf{9})^{20}$ e o diterpenoide $E$-fitol (11). ${ }^{22}$ A mistura M3 (Figura 20S) é formada pelos esteroides $3 \beta-\mathrm{OH}$ sitosterol (5, 47,31\%), estigmasterol $(\mathbf{6}, 25,15 \%)$, campesterol (12, 17,96\%), campestanol (13, 1,17\%), $\Delta^{22}$-stigmastenol $(\mathbf{1 4}, 2,44 \%)$, e sitostanol $(\mathbf{1 5}, 3,76 \%)^{21}$ e a mistura $M 4$ contém 7-cetositosterol (16) e 7-cetoestigmasterol (17). ${ }^{23}$ As estruturas dos compostos 1-11 e 16-20 foram identificadas pela análise dos espectros de RMN de ${ }^{1} \mathrm{H}$ e ${ }^{13} \mathrm{C}$ e comparação com dados da literatura. Os esteroides 12-15 e os ésteres metílicos dos ácidos graxos (a-e) foram identificados por comparação dos espectros de massas obtidos com os da biblioteca Wiley229. As estruturas dos compostos identificados são mostradas na Figura 1.

Não foram encontrados na literatura os dados de RMN para o composto 19. Seu espectro de RMN de ${ }^{1} \mathrm{H}$ (Figura 33S) apresentou somente dois simpletos em $\delta 5,85$ (H3/3') e 3,82 (H-5/5') com integração para um e três hidrogênios, respectivamente, sendo este último característico de grupo metoxila. O espectro de $\mathrm{RMN} \mathrm{de}{ }^{13} \mathrm{C}$ (Figura 34S) mostrou cinco sinais, sugerindo uma estrutura simétrica. Dois sinais em $\delta 187,0$ (C-4) e 176,8 (C-1/1') são de carbonilas, um em $\delta 157,5$ (C-2/2') é de carbono sp $^{2}$ não hidrogenado ligado a oxigênio, um em $\delta 107,6$ (C-3/3') de carbono metínico sp² e um em $\delta 56,2\left(\mathrm{C}-5 / 5^{\prime}\right)$ referente a metoxila. A análise do mapa de contornos $g \mathrm{HMBC}$ (Tabela 1 e Figura 36S) mostrou correlações de H-3/3' $(\delta 5,85)$ com C-4 $(\delta 187,0), \mathrm{C}-2 / 2^{\prime}(\delta 157,5)$ e C-1/1' $(\delta 176,8)$ e de H-5/5' $(\delta 3,82)$ com C-1/1' $(\delta 176,8)$ e C-2/2' $(\delta 157,5)$. A análise dos dados de RMN permitiram identificar o chelidonato de metila (19). Este composto é o éster metílico do ácido chelidônico, uma $\gamma$-pirona já relatada na família Rubiaceae. ${ }^{4,24} \mathrm{O}$ composto $\mathbf{1 9}$ possui atividade 


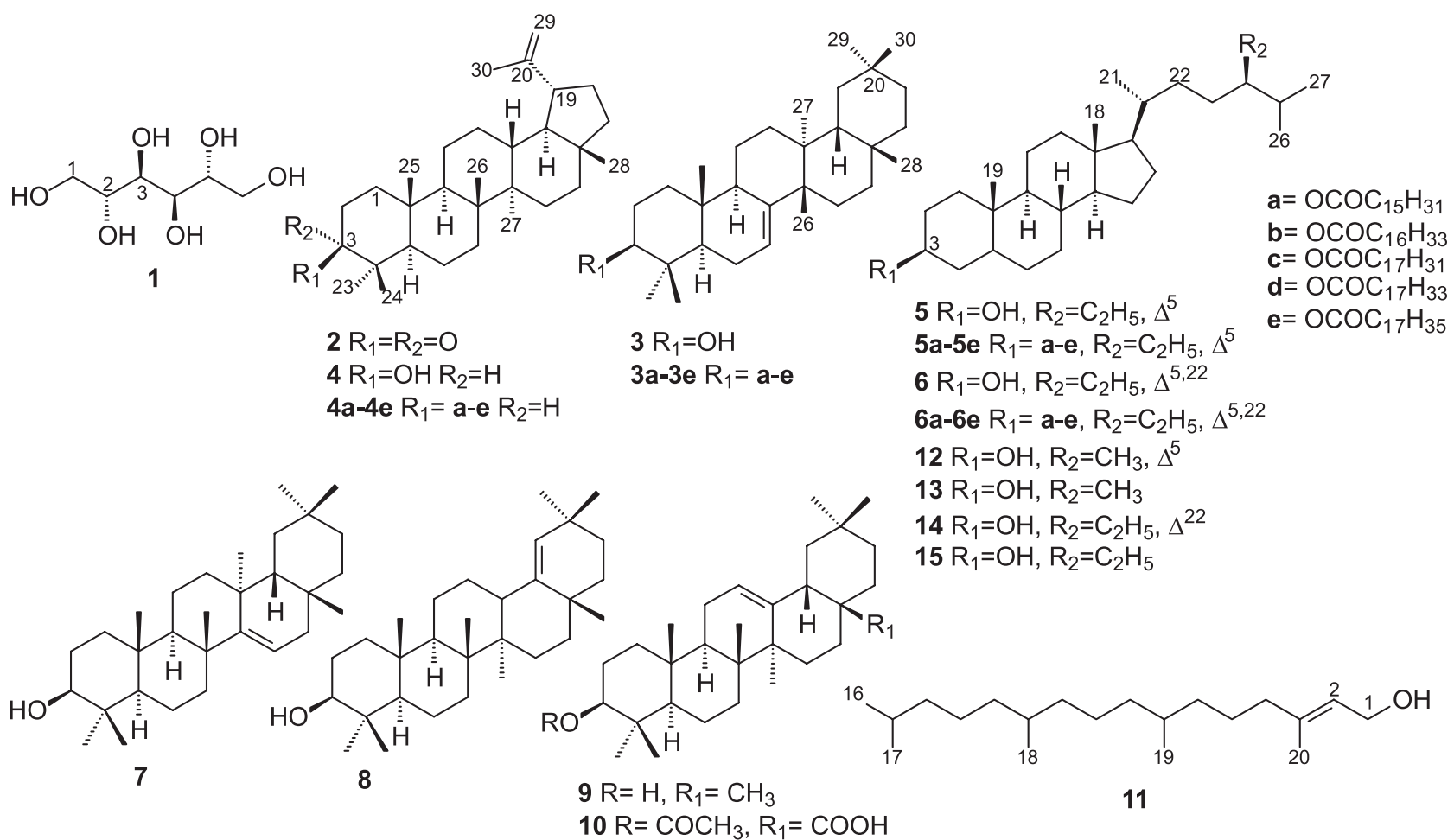<smiles>[Y6]CC12C(C(C)CCC(CC)C(C)C)CCC1C1(C)CCC3C(C(=O)C=C4CC(O)CCC43C)C12</smiles><smiles>CC(C)=CCC1=C(O)C(=O)c2ccccc2C1=O</smiles>

18<smiles>COC(=O)c1cc(=O)cc(C(=O)OC)o1</smiles>

19<smiles>O=C1OCC[C@H]2CC[C@H](CO)[C@H]12</smiles>

20

Figura 1. Constituintes químicos do caule de $\mathrm{T}$. hispidula

Tabela 1. Dados de RMN de ${ }^{1} \mathrm{H} \mathrm{e}{ }^{13} \mathrm{C}$ do chelidonato de metila (19) $\left(\mathrm{CDCl}_{3}\right.$, 400 e $100 \mathrm{MHz}, \delta$ em ppm)

\begin{tabular}{cccc}
\hline $\mathrm{C}$ & $\delta_{\mathrm{C}}$ & $\delta_{\mathrm{H}}$ & $g \mathrm{HMBC}$ \\
\hline $1 / 1^{\prime}$ & 176,8 & - & $\mathrm{H}-3\left({ }^{3} J\right), \mathrm{H}-5\left({ }^{3} J\right)$ \\
$2 / 2^{\prime}$ & 157,5 & - & $\mathrm{H}-3\left({ }^{2} J\right), \mathrm{H}-5\left({ }^{4} J\right)$ \\
$3 / 3^{\prime}$ & 107,6 & $5,85 \mathrm{~s}$ & - \\
4 & 187,0 & - & $\mathrm{H}-3\left({ }^{2} J\right)$ \\
$5 / 5$ & 56,6 & $3,82 \mathrm{~s}$ & - \\
\hline
\end{tabular}

anti-alérgica e foi relatado pela primeira vez de fonte natural em Senna spectabilis (Leguminosae). ${ }^{25}$

Com exceção do D-(+)-manitol (1), $\beta$-amirina (9), sitosterol (5) e estigmasterol (6), todos os demais compostos estão sendo relatados pela primeira fez no gênero Tocoyena. $\mathrm{O}$ palmitato e estearato de multiflorenila (3a e 3e), margarato, linoleato, oleato e estearato de lupeila (4b-4e), margarato, oleato e estearato de sitosterila $(\mathbf{5 b}$, $\mathbf{5 d}$ e 5e), oleato e estearato de estigmasterila (6d-6e) e chelidonato de metila (19) estão sendo relatados pela primeira vez na família Rubiaceae. O composto 20 é um 11-noriridoide, resultante da perda de C-11. Os derivados de 11-noriridoide-1,3-olídeo são raramente encontrados de fontes naturais, entretanto, foi relatado em Morinda officinalis (Rubiaceae). ${ }^{26}$

\section{Atividade antileishmania}

A leishmaniose é uma doença tropical negligenciada, causada por protozóarios do gênero Leishmania, os quais se apresentam nas formas promastigota (extracelular) e amastigota (intracelular). Clinicamente, a leishmaniose apresenta-se nas formas cutânea e mucocutânea resultando em lesões superficiais e, na forma visceral ataca os orgâos internos podendo levar a óbito. ${ }^{27}$

A ausência de vacinas e a toxicidade dos medicamentos atualmente usados para tratar a leishmaniose impulsiona a busca por novas substâncias ativas provenientes de plantas. ${ }^{27}$ Dessa forma, considerando a ocorrência de atividade antileishmania em plantas da família Rubiaceae, ${ }^{6}$ o extrato, frações e os compostos 19 e 20 de T. hipidula foram investigados frente a formas prosmatigostas de Leishmania major. A Tabela 2 mostra que o extrato $\mathrm{EtOH}$, as frações $\mathrm{CHCl}_{3}$, AcOEt e MeOH e os compostos 19 e 20 inibiram o crescimento de $L$. major. As frações $\mathrm{CHCl}_{3}\left(\mathrm{CI}_{50}=26,25 \mu \mathrm{g} \mathrm{mL}-1\right)$ e $\mathrm{AcOEt}\left(\mathrm{CI}_{50}=29,77\right.$ $\left.\mu \mathrm{g} \mathrm{mL} \mathrm{mL}^{-1}\right)$ mostraram-se mais ativas que a fração $\mathrm{MeOH}\left(\mathrm{CI}_{50}=247,34\right.$ $\left.\mu \mathrm{g} \mathrm{mL}{ }^{-1}\right)$ e o extrato $\mathrm{EtOH}\left(\mathrm{CI}_{50}=105,19 \mu \mathrm{g} \mathrm{mL}^{-1}\right)$, evidenciando que o fracionamento em coluna cromatográfica filtrante concentrou a atividade antileishmania nas frações $\mathrm{CHCl}_{3}$ e AcOEt e que a espécie possui compostos ativos com polaridades diferentes.

A atividade antileishmania da fração $\mathrm{CHCl}_{3}$ pode ser atribuída em parte ao chelidonato de metila (19) mas, sobretudo, a outros compostos isolados desta fração que já possuem atividade antileishmania 
descrita na literatura, como o lapachol $\left(\mathrm{CI}_{50}=5,2 \pm 0,7 \mu \mathrm{g} \mathrm{mL}{ }^{-1}\right),{ }^{28}$ ácido acetiloleanólico $\left(\mathrm{CI}_{50}=5,0 \pm 0,1 \mu \mathrm{M}\right),{ }^{29}$ lupeol $\left(\mathrm{CI}_{50}=39,06 \mu \mathrm{g} \mathrm{mL}^{-1}\right)^{30} \mathrm{e}$ mistura de sitosterol e estigmasterol $\left(\mathrm{CI}_{50}=70 \pm 0 \mu \mathrm{g} \mathrm{mL}-1\right),{ }^{31}$ os quais foram testados frente a L. amazonensis.

Tabela 2. Atividade antileishmania de T. hispidula frente a L. major

\begin{tabular}{lc}
\hline Amostras & $\mathrm{CI}_{50}$ em $\mu \mathrm{g} \mathrm{mL} \mathrm{L}^{-1}$ \\
\hline Extrato EtOH & 105,19 \\
Fração $\mathrm{CHCl}_{3}$ & 26,25 \\
Fração AcOEt & 29,77 \\
Fração MeOH & 247,34 \\
Chelidonato de metila (19) & 163,39 \\
Morindolídeo (20) & 424,20 \\
Anfotericina B (controle positivo) & 1,74 \\
\hline
\end{tabular}

\section{CONCLUSÃO}

O estudo fitoquímico da fração $\mathrm{CHCl}_{3}$ do extrato EtOH do caule de T. hispidula conduziu ao isolamento de seis compostos puros e quatro misturas, com predominância de triterpenoides e esteroides. Nos ensaios de atividade antileishmania frente a promastigotas de L. major as frações $\mathrm{CHCl}_{3}$ e AcOEt mostraram-se mais ativas que o extrato EtOH, fração $\mathrm{MeOH}$, chelidonato de metila (19) e morindolídeo (20). Os resultados obtidos contribuem para o conhecimento da composição química do extrato EtOH do caule de T. hispidula e demonstra o potencial antileishmania da espécie.

\section{MATERIAL SUPLEMENTAR}

Espectros de RMN de ${ }^{1} \mathrm{H} \mathrm{e}{ }^{13} \mathrm{C}$, infravermelho (IV), cromatogramas e espectros de massas dos compostos estão disponíveis em http:// quimicanova.sbq.org.br, na forma de arquivo PDF, com acesso livre.

\section{AGRADECIMENTOS}

Os autores agradecem à Capes, ao CNPq e ao CNPq/INCTBioNat (465637/2014-0) pelo apoio financeiro e bolsas de E. A. Sousa e M. H. Chaves (302639/2015-2). Ao LAUREMN/UFPI pelos espectros de RMN e à Dra. R. R. S. Farias pela coleta e identificação do material vegetal.

\section{REFERÊNCIAS}

1. Delprete, P. G.; Jardim, J. G.; Rodriguésia 2012, 63, 101.

2. Barbosa, M. R.; Zappi, D.; Taylor, C.; Cabral, E.; Jardim, J. G.; Pereira, M. S.; Calió, M. F.; Pessoa, M. C. R.; Salas, R.; Souza, E. B.; Di Maio, F. R.; Macias, L.; Anunciação, E. A.; Germano Filho, P.; Oliveira, J. A.; Bruniera, C. P.; Gomes, M.; De Toni, K.; Firens, M.; 2015 Rubiaceae in Lista de Espécies da Flora do Brasil. Jardim Botânico do Rio de Janeiro. Disponível em: http://floradobrasil.jbrj.gov.br/jabot/ floradobrasil/FB210, acessado em novembro 2018.

3. Bolzani, V. S.; Young, M. C. M.; Furlan, M.; Cavalheiro, A. J.; Araújo, A. R.; Silva, D. H. S.; Lopes, M. N.; Recent Res. Dev. Phytochem. 2001, 5,19 .

4. Martins, D.; Nunez, C. V.; Molecules 2015, 20, 13422.

5. Haudecoeura, R.; Peuchmaura, M.; Pérèsa, B.; Rome, M.; Taïwe, G. S.; Boumendjela, A.; Boucherle, B.; J. Ethnopharmacol. 2018, 212, 106; Taika, B. B.; Bouckandou, M.; Souza, A.; Bouroboub, H. P. B.; MacKenzie, L. S.; Lione, L.; J. Ethnopharmacol. 2018, 216, 203; Assi, R. A.; Darwis, Y.; Abdulbaqi, I. M.; khan, L. V.; Vuanghao, L.; Laghari, M. H.; Arabian J. Chem. 2017, 10, 691.
6. Rocha, L. G.; Almeida, J. R. G. S.; Macêdo, R. O.; Barbosa-Filho, J. M.; Phytomedicine 2015, 12, 514; Moreira, V. F.; Vieira, I. J. C.; Braz-Filho, R.; Am. J. Plant Sci. 2015, 6, 2612; Kato, L.; Oliveira, C. M. A.; Faria, E. O.; Ribeiro, L. C.; Carvalho, B. G.; Silva, C. C.; Schuquel, I. T. A.; Santin, S. M. O.; Nakamura, C. V.; Britta, E. A.; Miranda, N.; Iglesias, A. H.; Delprete, P. G.; J. Braz. Chem. Soc. 2012, 23, 355; Baldé, E. S.; Megalizzi, V.; Traoré, M. S.; Cos, P.; Maes, L.; Decaestecker, C.; Pieters, L.; Baldé, A. M.; J. Ethnopharmacol. 2010, 130, 529; Ahua, K. M.; Ioset, J.-R.; Ioset, K. N.; Diallo, D.; Mauel, J.; Hostettmann. K.; J. Ethnopharmacol. 2007, 110, 99.

7. Oliveira, J. A.; 2015. Tocoyena in Lista de espécies da Flora do Brasil. Jardim Botânico do Rio de Janeiro. Disponível em: http://floradobrasil. jbrj.gov.br/jabot/floradobrasil/FB14335, acessado em novembro 2018.

8. Poser, G. L. V.; Seibt, L. T.; Biochem. Syst. Ecol. 1998, 26, 669.

9. Bolzani, V. S.; Izumisawa, C. M.; Young, M. C. M.; Trevisan, L. M. V.; Kingston, D. G. I.; Phytochemistry 1997, 46, 305.

10. Hamerski, L.; Carbonezi, C. A.; Cavalheiro, A. J.; Bolzani, V. S.; Young, M. C. M.; Quim. Nova 2005, 28, 601. Bolzani, V. S.; Trevisan, L. M. V.; Izumisawa, C. M.; Young, M. C. M.; J. Braz. Chem. Soc. 1996, 7, 157; Santos, W. P.; Santos, H. S.; Bandeira, P. N.; Pessoa, O. D. L.; Silveira, E. R.; Braz-Filho, R.; Albuquerque, M. R. J. R.; Resumos da $34^{\circ}$ Reunião Anual da Sociedade Brasileira de Química, Florianópolis, Brasil, 2011; Rocha, M. O.; Dissertação de Mestrado, Universidade Federal de Alagoas, Brasil, 2009.

11. Cesário, F. R. A. S.; Albuquerque, T. R.; Lacerda, G. M.; Oliveira, M. R. C.; Silva, B. A. F.; Rodrigues, L. B.; Martins, A. O. B. P. B.; Almeida, J. R. G. S.; Vale, M. L.; Coutinho, H. D. M.; Menezes, I. R. A.; Saudi J. Biol. Sci. (2018), doi:10.1016/j.sjbs.2018.01.008; Cesário, F. R. A. S.; Albuquerque, T. R.; Lacerda, G. M.; Oliveira, M. R. C.; Rodrigues, L. B.; Martins, A. O. B. P. B.; Boligon, A. A.; Quintans-Júnior, L. J. Q.; Araújo, A. A. S.; Vale, M. L.; Coutinho, H. D. M.; Menezes, I. R. A.; Biomed. Pharmacother. 2018, 97, 321; Barros, G. S. G.; Matos, F. J. A.; Vieira, J. E. V.; Sousa, M. P.; Medeiros, M. C.; J. Pharm. Pharmacol. 1970, 22, 116; Cesário, F. R. A. S.; Albuquerque, T. R.; Silva, B. A. F.; Lacerda, G. M.; Rodrigues, L. B.; Martins, A. O. B. P. B.; QuintansJúnior, L. J.; Almeida, J. R. G. S.; Vale, M. L.; Coutinho, H. D. M.; Menezes, I. R. A.; Food Chem. Toxicol. 2018, 112, 355; Ribeiro, D. S. F.; Freire, J. M. O.; Teixeira, A. H.; Val, D. R.; Freitas, A. R.; Gomes, F. I. F.; Silva, A. A. R.; Bandeira, P. N.; Santos, H. S.; Santos, W. P.; Ávila, F. N.; Pereira, K. M. A.; Goes, P.; Pinto, V. P. T.; Cristino-Filho, G.; Albuquerque, M. R. J. R.; Chaves, H. V.; Bezerra, M. M.; Biomed. Pharmacother. 2018, 98, 863.

12. Silva, M. P.; Dissertação de Mestrado, Universidade Federal do Piauí, Brasil, 2010; Oliveira, L. S. D.; Soares, S. M. N. A.; Soares, F. A. R.; Barros, R. F. M.; Revista Brasileira de Biociências 2007, 5, 372.

13. Silva, H. R.; Silva, C. C. M.; Caland-Neto, L. B; Lopes, J. A. D.; Citó, A. M. G. L.; Chaves, M. H.; Quim. Nova 2007, 30, 1877.

14. Valadares, D. G.; Duarte, M. C.; Oliveira, J. S.; Chávez-Fumagalli, M. A.; Martins, V. T.; Costa, L. E.; Leite, J. P. V.; Santoro, M. M.; Régis, W. C. B.; Tavares, C. A. P.; Coelho, E. A. F.; Parasitol. Int. 2011, 60, 357; Soares, D. C.; Pereira, C. G.; Meireles, M. A. A.; Saraiva, E. M.; Parasitol. Int. 2007, 56, 135.

15. Paula, V. F.; Barbosa, L. C. A; Piló-Veloso, D.; Demuner, A. J.; Howarth, O.; Ecletica Quim. 1998, 23, 45.

16. Olea, R. S. G.; Roque, N. F.; Quim. Nova 1990, 13, 278.

17. Jo, Y.; Suh, J.; Shin, M. H.; Jung, J. H.; Im, K. S.; Arch. Pharmacal Res. 2005, 28, 885 .

18. Moreira, R. Y. O.; Arruda, M. S. P.; Arruda, A. C.; Santos, L. S.; Müller, A. H.; Guilhon, G. M. S. P.; Santos, A. S.; Terezo, E.; Rev. Bras. Farmacogn. 2006, 16, 392.

19. Yoshikawa, M.; Yamaguchi, S.; Nishisaka, H.; Yamahara, J.; Murakami, N.; Chem. Pharm. Bull. 1995, 43, 1462.

20. Mahato, S. B.; Kundu, A. P.; Phytochemistry 1994, 37, 1517. 
21. De-Eknamkul, W.; Potduang, B.; Phytochemistry 2003, 62, 389.

22. Rahman, A.; Ahmad, V. U. ${ }^{13}$ C-NMR of Natural Products: Diterpenes. Plenum Press: New York, 1992.

23. Wang, Q.-Y.; Cui, G.-X.; Wu, J.-C.; Chen, Y.-G.; Chem. Nat. Compd. 2015, 51, 1196; Li, R.-J.; Guo, D.-X.; Lou, H.-X.; Chin. J. Nat. Med. 2013, $11,74$.

24. Shen, Z.-W.; Fisinger, U.; Poulev, A.; Eisenreich, W.; Werner, I.; Pleiner, E.; Bacher, A.; Zenk, M. H.; Phytochemistry 2001, 57, 33.

25. Mallaiah, B. V.; Kumar, K. A.; Sarma, P. N.; Srimannarayana, G.; Curr. Sci. 1984, 53, 33.

26. Ban, N. K.; Giang, V. H.; Linh, T. M.; Lien, L. Q.; Ngoc, N. T.; Thao, D. T.; Nam, N. H.; Cuong, N. X.; Kiem, P. V.; Minh, C. V.; Phytochem. Lett. 2013, 6, 267.

27. Cock, I. E.; Selesho, M. I.; Vuuren, S. F. V.; J. Etnopharmacol. 2018 , $220,250$.
28. Lima, N. M. F.; Correia, C. S.; Leon, L. L.; Machado, G. M. C.; Madeira, M. F.; Santana, A. E. G.; Goulart, M. O. F.; Mem. Inst. Oswaldo Cruz 2004, 99, 757.

29. Gnoatto, S. C. B.; Vechia, L. D.; Lencina, C. L.; Dassonville-Klimpt, A.; Nascimento, S.; Mossalayi, D.; Guillon, J.; Gosmann, G.; Sonnet, P.; J. Enzyme Inhib. Med. Chem. 2008, 23, 604.

30. Souza, A. C.; Alves, M. M. M.; Brito, L. M.; Oliveira, L. G. C.; Sobrinho-Júnior, E. P. C.; Costa, I. C. G.; Freitas, S. D. L.; Rodrigues, K. A. F.; Chaves, M. H.; Arcanjo, D. D. R.; Carvalho, F. A. A.; J. Evidence-Based Complementary Altern. Med. 2017, 2017, 1.

31. Silva, A. A. S.; Morais, S. M.; Falcão, M. J. C.; Vieira, I. G. P.; Ribeiro, L. M.; Viana, S. M.; Teixeira, M. J.; Barreto, F. S.; Carvalho, C. A.; Cardoso, R. P. A.; Andrade-Junior, H. F.; Phytomedicine 2014, 21, 1419. 